\title{
Sufficient conditions for the prediction-based stabilization of linear systems subject to input with input-varying delay
}

\author{
Delphine Bresch-Pietri, Jonathan Chauvin, Nicolas Petit
}

\begin{abstract}
This paper contains a result proving that a predictor feedback controller can effectively yield asymptotic convergence for a class of linear systems with input-dependent delay. The delay is implicit and its model involves past values of the input. It is representative of systems where transport phenomena take place. This situation is frequent in the process industry. The conditions on asymptotic stabilization require the feedback gain to be small. Arguments of proof for this novel result include general Halanay inequalities for delay differential equations and build on recent advances of backstepping techniques for uncertain or varying delay systems.
\end{abstract}

\section{INTRODUCTION}

Numerous control systems involve a physical dead-time which reveals troublesome in the design and tuning of feedback control laws. In practice, such dead-time occur, e.g., when sensors and actuators are not co-located. Prime examples are processes involving transportation of material, such as mixing processes for liquid or gaseous fluids, chemical reactors [10], automotive engine and exhaust line [7], heat collector plant [22], blending in liquid or solid networks [6], and batch processes [20], to name a few. Remarkably, in all these examples, the lag directly depends on the control variable and the considered delay is inherently inputdependent (see the example of the crushing-mill presented in [21]).

Surprisingly, it seems that stabilization of such processes with input-dependent time-delay in the input $D(u)$ or $D\left(u_{t}\right)$, where $u_{t}$ denotes past values over a finite horizon, has seldom been theoretically studied. Rather, a widely considered approach is to recast the delay dependence on the control, e.g. by modeling this dependence as $D(u) \approx D(t)$ (or even by a constant average value $D$ ) and by asking the controller to deal with a certain level of unstructured model variability.

Predictor-based control strategies (see [23], [1], [15]), which are state-of-the-art for systems with constant input time-delays (see for instance [8], [12], [16], [3], [17] or [21] and the reference therein) are still not of general use for time-varying delays. In [18] or, more recently, in [14], a time-varying delay version of predictor-based control has been presented. To compensate the input delay, the prediction is calculated over a time window of which length matches the value of the future delay. In other words, one needs to

D. Bresch-Pietri (corresponding author) is with the Department of Mechanical Engineering, MIT, Cambridge, MA 02139, USA Email: dbp@mit.edu

J. Chauvin is with the Département Contrôle, Signal et Système in IFP Energies nouvelles, 1-4 Av. du Bois Préau, 92852 Rueil Malmaison, France

N. Petit is with the Centre Automatique et Systèmes, Unité Mathématiques et Systèmes at MINES ParisTech, 60 Bd St Michel, 75272 Paris, France predict the future variations of the delay. For example, this is the approach followed in [24] for a time-varying delay, the variations of which are provided by a given known model. It has also been used in [2] for a state-dependent delay, where variations are characterized by a careful prediction of the system state.

Yet, when the delay depends on the input, things are getting very involved. Determining the required prediction horizon becomes an implicit issue, which may not be practically solvable nor even well-posed. This implicit nature is caused by the reciprocal interactions between the control (current and past) values and the delay, yielding a closedloop dependency.

In this paper, a prediction technique is also used. We blend the previously described techniques and establish sufficient conditions for asymptotic stabilization using a two-steps methodology enabling to disrupt the implicit loop.

In a first move, we consider the input-dependency as a particular type of time-variations. It enables then to use a robust compensation result which has been obtained for linear systems with time-varying input delay, using the backstepping tools proposed in [13] for the analysis of inputdelay systems stability. Technically, this result guarantees stabilization provided the delay variations are sufficiently small. In a second step, a sufficient bound on the delay variations is obtained by relating them to the control tracking error, which is analyzed using the asymptotic convergence of delay differential equations (DDE) (Halanay-type inequalities [9]).

To the best of the authors' knowledge, this connection between prediction-based control law and input-dependency of the delay has not been studied earlier (except on preliminary works for a scalar system studied by the authors in [4] ). The result that we establish for a potentially unstable systems of order $n$ with a particular model of input-dependent delay is the main contribution of the paper.

The considered integral relation defining the delay through past values of the input is representative of a large class of flow processes. For this class of systems robust compensation is shown to be achievable, provided that that initial conditions are close enough to equilibrium and that the feedback gain is chosen accordingly.

The paper is organized as follows. In Section II, we describe the problem under consideration. We design a prediction-based controller in Section III. Sufficient conditions for stabilization are derived through a proof of convergence invoking Halanay-like inequalities arguments in Section IV. Before providing directions of future work, we 
detail an illustrative example in Section V.

\section{Notations and definitions}

In the following, |.| stands for the usual Euclidean norm, $\mathcal{C}^{0}\left(S_{1}, S_{2}\right)$ denotes the set of continuous functions on the set $S_{1}$ with values into the set $S_{2}$. The operator Sat ${ }_{I}$ stands for the standard saturation operator onto the interval $I$.

For a given symmetric matrix, $\underline{\lambda}($.$) and \bar{\lambda}($.$) stands$ respectively for minimum and maximum eigenvalues.

$x_{t}$ refers to the function $x_{t}: s \in[-\bar{D}, 0] \mapsto x(t+s)$ for a given function $x$ and $\bar{D}>0$.

For any bounded function $k$ defined on $[-\bar{D}, 0]$, a polynomial function $\pi\left(x\left(t_{1}\right), \ldots, x\left(t_{n-2}\right), \int_{t_{n-1}}^{t_{n}} k(t-s) x(s) d s\right)$ for $\left(t_{1}, \ldots, t_{n}\right) \in[t-\bar{D}, t]^{n}$ is denoted $\pi\left(x_{t}\right)$.

A polynomial function $\pi$ in the variables $\left(x_{1}, \ldots, x_{n}, x_{n+1}\right)$ is said to be at least quadratic in $x_{1}, \ldots, x_{n}$ iff, for any given $x_{n+1}$, the corresponding polynomial function $\pi_{x_{n+1}}$ defined as

$$
\pi_{x_{n+1}}\left(x_{1}, \ldots, x_{n}\right)=\pi\left(x_{1}, \ldots, x_{n}, x_{n+1}\right)
$$

has no terms of order 0 or 1 , e.g. $\pi=x_{1}^{2}+x_{1} x_{2} x_{3}$ and $\pi=x_{2} x_{1}+x_{3} x_{1}^{2}$ are both at least quadratic in $\left(x_{1}, x_{2}\right)$ while $\pi=x_{3}+x_{3} x_{2}^{2}$ is not.

\section{Problem Statement}

Consider the following potentially unstable linear timeinvariant plant ${ }^{1}$ sketched in Fig. 1

$$
x^{(n)}+a_{n-1} x^{(n-1)}+\ldots+a_{1} \dot{x}+a_{0} x=b_{0} \phi(t-D(t))
$$

driven by a delayed input, where the varying delay $D(t)$ is implicitly defined in terms of the input history by

$$
\int_{t-D(t)}^{t} \phi(s) d s=1 \quad \text { with } \quad \phi(t)=\operatorname{Sat}_{[\underline{u},+\infty[}(u(t)), \quad \underline{u}>0
$$

Physically, the integral equation (2) corresponds to a PlugFlow assumption [19] in a transport phenomenon and appears in a large class of applications where $\phi$ is a (normalized) flow rate. Because $\phi \geq \underline{u}>0$, this transport delay is well defined ${ }^{2}$ and is upper-bounded: $D(t) \leq \bar{D} \triangleq 1 / \underline{u}, t \geq 0$. In particular, as $D \mapsto \int_{t-D}^{t} \phi(s) d s$ is strictly increasing, it is invertible and the delay can be calculated and is therefore assumed to be known in the following (see Section V for details).

The control objective is to stabilize the plant over any equilibrium point such that $x^{r}=b_{0} / a_{0} u^{r}$ with $u^{r} \geq \underline{u}$. For this purpose, a predictor-based feedback law will be employed here.

With this aim in view, we first formulate a state-space representation of this system as

$$
\left\{\begin{array}{l}
\dot{X}=A X(t)+B \phi(t-D(t)) \\
\int_{t-D(t)}^{t} \phi(s) d s=1 \quad \text { with } \phi(t)=\operatorname{Sat}_{[\underline{u},+\infty[}(u(t))
\end{array}\right.
$$

\footnotetext{
${ }^{1}$ Potential existing zeros can still be handled by a suitable aforementioned choice of state-space representation and of the output matrix.

${ }^{2}$ The delay is positive and, besides, its derivative can be expressed as $\dot{D}=1-\frac{\phi(t)}{\phi(t-D)}<1$ which guarantees strict causality.
}

where

$$
A=\left(\begin{array}{cccc}
0 & 1 & & 0 \\
\vdots & & \ddots & \\
0 & 0 & & 1 \\
-a_{0} & -a_{1} & \ldots & -a_{n-1}
\end{array}\right), \quad B=\left(\begin{array}{c}
0 \\
\vdots \\
0 \\
b_{0}
\end{array}\right)
$$

For sake of clarity, in the following we assume that the system state $X$ is fully measured. Extension to observable systems could be considered in future works. To construct a prediction-based control law, we use the following theorem, a proof of which is given in [4] and [5].

Theorem 1: Consider the closed-loop single input system

$$
\begin{aligned}
\dot{X}(t) & =A X(t)+B u(t-D(t)) \\
u(t) & =K\left[e^{A D(t)} X(t)+\int_{t-D(t)}^{t} e^{A(t-s)} B u(s) d s\right]
\end{aligned}
$$

where $X \in \mathbb{R}^{n}, u \in \mathbb{R}, K$ is chosen such that $A+B K$ is Hurwitz and $D: \mathbb{R}_{+} \rightarrow[0, \bar{D}]$ is a time-differentiable function. There exists $\left.\Delta^{*}(|K|) \in\right] 0 ; 1[$ such that, provided

$$
\forall t \geq 0, \quad|\dot{D}(t)|<\Delta^{*}(|K|)
$$

the plant (4a) exponentially converges to the origin.

The prediction controller (4b) is a natural extension from the case of constant delay. It forecasts values of the state over a time window of varying length $D(t)$. Of course, exact compensation of the delay is not achieved with this controller. To do so, one would need to consider a time window of which length would exactly match the value of the future delay, as it is made in [18] and [14]. In details, defining $\eta(t)=t-D(t)$ and assuming that its inverse exists ${ }^{3}$, exact delay-compensation is obtained with the feedback law $U(t)=K X\left(\eta^{-1}(t)\right)$. Yet, implementing this relation requires to predict the future variation of the delay via $\eta^{-1}(t)$, which is not practically achievable for an input-varying delay.

Equation (5) can be interpreted as a condition for robust delay compensation achievement ${ }^{4}$. The spirit of this condition is that, if the delay varies sufficiently slowly, its current value $D(t)$ used for prediction remains close enough to its future values, and the corresponding prediction is accurate enough to guarantee the stabilization of the plant through the feedback loop.

We now focus on an alternative sufficient condition guaranteeing (5) when the delay varies according to the integral relation (2). For practical control design, this new condition bears on the control gain and the initial conditions.

\footnotetext{
${ }^{3}$ which is the case if $\dot{D}<1$

${ }^{4}$ Interestingly, a similar condition is often stated in Linear Matrix Inequality approaches, such as [25] for example, where the delay is also assumed to be time-differentiable.
} 


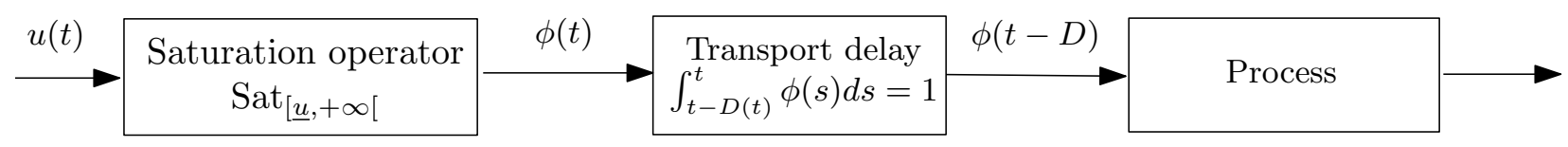

Fig. 1. The addressed problem, where the input is delayed by a transport delay which is input-varying.

\section{Control Design}

Theorem 2: Consider the closed-loop system

$$
\left\{\begin{array}{l}
\dot{X}(t)=A X(t)+B \phi(t-D(t)) \\
\int_{t-D(t)}^{t} \phi(s) d s=1 \quad \text { with } \phi(t)=\operatorname{Sat}_{[\underline{u},+\infty[}(u(t)) \\
u(t)=u^{r}+K\left[e^{A D(t)} X(t)+\int_{t-D(t)}^{t} e^{A(t-s)} B \phi(s) d s-X^{r}\right]
\end{array}\right.
$$

where $A$ and $B$ are defined in (3), $K$ is such that $A+B K$ is Hurwitz, $U$ is scalar, $X^{r}$ is the state equilibrium corresponding to the original equilibrium $x^{r}$ of plant (1) and $U^{r}$ is the corresponding (constant) reference control. Consider the functional

$$
\Theta(t)=\left|X(t)-X^{r}\right|+\max _{s \in[t-\bar{D}, t]}\left|u(s)-u^{r}\right|
$$

Then, there exists $\theta: \mathbb{R}^{n} \mapsto \mathbb{R}_{+}^{\star}$ such that is $\Theta(0)<\theta(K)$ the condition (5) is fulfilled and the plant exponentially converges to $X^{r}$.

This result has a relatively direct interpretation: the previously presented Theorem 1 requires the delay to vary sufficiently slowly, while, on the other hand, the delay variations implicitly depend on the control input through the integral equation (2), with variations which aggressiveness is scaled by the gain $K$. Then, restricting the input variations by choosing the initial conditions close enough to the desired equilibrium and in compliance with the feedback gain magnitude seems like a natural requirement.

The behavior of the function $\theta$ with respect to $K$ should be investigated in future works. From the expression given in (16), it would involve to study the solution of the Lyapunov equation and the behavior of its eigenvalues with respect to $K$. One could reasonably that $\theta(K) \rightarrow 0$ while $K \rightarrow-\infty$ (as $r(K)$ is expected in this case to tend to $\infty$ ). Therefore, for given initial conditions, the magnitude of the feedback gain should be chosen accordingly. For relatively large initial conditions, this would imply to use a small feedback gain. Therefore, this result can be interpreted as a small-gain condition.

We now detail the proof of Theorem 2 .

\section{Proof of Theorem 2 - Halanay-Like INEQUALITIES}

Taking a time-derivative of (7) and defining the error variable

$$
\varepsilon \triangleq
$$

one gets, using $\phi \geq \underline{u}$,

$$
\dot{D}(t)=1-\frac{\varepsilon(t)+u^{r}}{\varepsilon(t-D(t))+u^{r}} \leq \frac{2 \max \left|\varepsilon_{t}\right|}{\underline{u}}
$$

As a result, condition (5) is satisfied if

$$
\forall t \geq 0, \quad \max \left|\varepsilon_{t}\right|<\frac{\underline{u} \Delta^{*}(|K|)}{2}
$$

This is the condition we now focus on. It yields to the analysis of the dynamics of the variable $\varepsilon$. Before properly starting this analysis, we recall and extend some well-known stability results for DDE.

A. Preliminary results : extension of the Halanay inequality for Delay Differential Equations of order $n \geq 1$

We first recall the following result ([9], [11] $)^{5}$.

Lemma 1: (Halanay inequality) Consider a positive continuous real-valued function $x$ such that, for some $t_{0} \in \mathbb{R}$,

$$
\dot{x}(t) \leq-a x(t)+b \max x_{t}, \quad t \geq t_{0}
$$

with $a \geq b \geq 0$. Then, there exists $\gamma \geq 0$ such that

$$
\forall t \geq t_{0}, \quad x(t) \leq \max x_{t_{0}} e^{-\gamma\left(t-t_{0}\right)}
$$

A straightforward extension of this lemma is stated in the corollary below.

Corollary 1: Consider a delay differential equation of the form

$$
\left\{\begin{aligned}
\dot{x}(t) & +a x(t)+b h\left(t, x_{t}\right)=0, \quad t \geq t_{0} \\
x_{t_{0}} & =\psi \in \mathcal{C}^{0}([-\bar{D}, 0], \mathbb{R})
\end{aligned}\right.
$$

where $h$ is a continuous functional satisfying, on an open neighborhood $\Omega$ of the origin, the sup-norm relation

$$
\left|h\left(t, x_{t}\right)\right| \leq \max \left|x_{t}\right|, \quad x_{t}:[-\bar{D}, 0] \rightarrow \Omega
$$

Then, if the initial condition $\psi$ has values into $\Omega$ and if $a \geq b \geq 0$, then there exists $\gamma \geq 0(\gamma=0$ if $a=b$ and $\gamma>0$ otherwise) such that every solution satisfies

$$
\forall t \geq t_{0}, \quad|x(t)| \leq \max \left|x_{t_{0}}\right| e^{-\gamma\left(t-t_{0}\right)}
$$

Proof: A proof is this corollary can be found in [4].

Lemma 2: [stability of a $n^{\text {th }}$ order DDE] Let $x$ be a solution of the $n^{\text {th }}$ order DDE

$$
\left\{\begin{array}{l}
x^{(n)}+\alpha_{n-1} x^{(n-1)}+\ldots+\alpha_{0} x=c \ell\left(t, x_{t}, \ldots x_{t}^{(n-1)}\right), t \geq t_{0} \\
X_{t_{0}}=\psi \in \mathcal{C}^{0}([-\bar{D}, 0], \mathbb{R}) \quad \text { with } \quad|\psi| \in \Omega
\end{array}\right.
$$

where the left-hand side of the differential equation defines a polynomial which roots have only strictly negative real parts, $c>0, \ell$ is a continuous functional, $\Omega=]-\underline{\omega}, \bar{\omega}[$ is

\footnotetext{
${ }^{5}$ More precisely, in [9], this result is stated for $a>b>0$.
} 
a neighborhood of the origin included inside a second one $\left.\Omega_{0}=\right]-\underline{\omega}_{0}, \bar{\omega}_{0}$ [ on which $\ell$ satisfies the sup-norm relation

$$
\forall t \geq t_{0}, \quad\left|\ell\left(t, x_{t}, \ldots, x_{t}^{(n-1)}\right)\right| \leq \max \left|X_{t}\right|
$$

with $\quad X=\left[\begin{array}{ll}x & \dot{x} \ldots x^{(n-1)}\end{array}\right]^{T}, \quad \underline{\omega}_{0}>\sqrt{\underline{\lambda}(P)} \underline{\omega}>0$, $\bar{\omega}_{0}>\sqrt{\underline{\lambda}(P)} \bar{\omega}>0$ and $P$ the solution of the Lyapunov equation associated to (14) for a given positive definite matrix $Q$. Then, there exists $c_{\max }>0$ such that, for any $0 \leq c<c_{\max }$, there exists $\gamma \geq 0$ and $r \geq 1\left(r=1, c_{\max }=\alpha_{0}\right.$ if $n=1)$ such that

$$
\forall t \geq 0, \quad|X(t)| \leq r \max \left|X_{t_{0}}\right| e^{-\gamma\left(t-t_{0}\right)}
$$

Proof: The idea is to use the scalar result of Corollary 1. Define the scalar valued function $m(t)=X^{T} P X$ where $P$, as defined in the Lemma, is the symmetric positive definite matrix solution of the Lyapunov equation $A_{0}^{T} P+P A_{0}=-Q$, for some given symmetric positive definite matrix $Q$ and $A_{0}$ the companion matrix

$$
A_{0}=\left(\begin{array}{cccc}
0 & 1 & & \\
\vdots & & \ddots & \\
0 & & & 1 \\
-\alpha_{0} & -\alpha_{1} & \ldots & -\alpha_{n-1}
\end{array}\right)
$$

Taking a time-derivative of $m$, one can obtain

$$
\begin{aligned}
\dot{m}(t) & =-X^{T}(t) Q X(t)+2 X(t)^{T} P\left(\begin{array}{c}
0 \\
\vdots \\
0 \\
c \ell\left(t, x_{t}, \ldots, x_{t}^{(n-1)}\right)
\end{array}\right) \\
& \leq-\frac{\lambda}{\overline{\bar{\lambda}}(P)} m(t)+2 c \bar{\lambda}(P)|X(t)|\left|\ell\left(t, x_{t}, \ldots, x_{t}^{(n-1)}\right)\right|
\end{aligned}
$$

Then,

$$
\dot{m}(t)+\frac{\underline{\lambda}(Q)}{\bar{\lambda}(P)} m(t) \leq \frac{2 c \bar{\lambda}(P)}{\sqrt{\underline{\lambda}(P)}} \sqrt{m(t)}\left|\ell\left(t, x_{t}, \ldots, x_{t}^{(n-1)}\right)\right|
$$

Define $a \triangleq \frac{\lambda}{\bar{\lambda}(P)}, b \triangleq 2 c \frac{\bar{\lambda}(P)}{\underline{\lambda}(P)}$ and

$$
h\left(t, m_{t}, \ldots, m_{t}^{(n-1)}\right) \triangleq \sqrt{\underline{\lambda}(P)} \sqrt{m(t)}|\ell|
$$

which satisfies over the neighborhood $\Omega_{0}$

$$
\left|h\left(t, m_{t}, \ldots, m_{t}^{(n-1)}\right)\right| \leq \sqrt{m(t)} \max \sqrt{m_{t}} \leq \max m_{t}
$$

Applying Corollary 1 , one concludes that if $m_{t_{0}}$ has values into $\Omega_{0}$ (which is the case as $X_{t_{0}}$ has values inside $\Omega$ ) and if $a>b$ then there exists $\gamma>0$ such that

$$
\forall t \geq t_{0}, \quad m(t) \leq \max m_{t_{0}} e^{-2 \gamma\left(t-t_{0}\right)}
$$

or

$$
\forall t \geq t_{0}, \quad|X(t)| \leq \sqrt{\frac{\bar{\lambda}(P)}{\underline{\lambda}(P)}} \max \left|X_{t_{0}}\right| e^{-\gamma\left(t-t_{0}\right)}
$$

Finally, the condition $a>b$ can be reformulated as $c<\frac{\underline{\lambda}(P) \underline{\lambda}(Q)}{2 \bar{\lambda}(P)^{2}}=c_{\max }$ which concludes the proof.
B. Application to the dynamical equation of $\varepsilon$ defined through the predictor-based control law (8)

We now focus on the DDE governing $\varepsilon$, which is given in the following lemma.

Lemma 3: Consider $t_{0} \in \mathbb{R}$ and assume that the function $\phi$ is unsaturated for $t \leq t_{0}$ (or equivalently that $u(t) \geq \underline{u}, t \leq t_{0}$ ). Then, the error variable $\varepsilon=u-u^{r}$ with $u$ defined in (8) satisfies the following differential equation for $t \leq t_{0}$

$$
\begin{aligned}
\varepsilon^{(n)}+\left(a_{n-1}\right. & \left.+b_{0} k_{n-1}\right) \varepsilon^{(n-1)}+\ldots+\left(a_{0}+b_{0} k_{0}\right) \varepsilon \\
& =\pi_{1}\left(\dot{D}, \ldots, D^{(n)}, \varepsilon_{t}, \ldots, \varepsilon_{t}^{(n-1)}, \frac{1}{1+\dot{D}}\right)
\end{aligned}
$$

where the constants $\left[-k_{0} \ldots-k_{n-1}\right] \stackrel{\Delta}{=} K$ and $\pi_{1}$ is a polynomial function which is at least quadratic in the variables $\varepsilon_{t}, \ldots, \varepsilon_{t}^{(n-1)}, \dot{D}, \ldots, D^{(n)}$.

Proof: For sake of clarity, the proof of this theorem is given in Appendix.

Now that we are equipped with this last lemma, it is possible to use Lemma 2 to guarantee that the stability condition (10) holds.

Lemma 4: Consider the functional $\Theta$ defined in (9). Then, there exists $\theta: \mathbb{R}^{n} \mapsto \mathbb{R}_{+}^{*}$ such that, if $\Theta(0)<\theta(K)$, then

$$
\forall t \geq 0, \quad|\varepsilon(t)| \leq \min \left\{\frac{\underline{u} \Delta^{\star}(|K|)}{2}, u^{r}-\underline{u}\right\}
$$

which implies that condition (10) is fulfilled.

Proof: Assume for a moment that the function $\phi$ is not saturated for $t \leq 0$. Then, dynamics (15) holds and is compliant with the assumptions of Lemma 2.

In details, first, the left-hand side of (15) is stable, as it represents the last line of the Hurwitz companion matrix $A+B K$. Second, by observing that

$$
\dot{D}=\frac{\varepsilon(t-D)-\varepsilon(t)}{\varepsilon(t-D)+u^{r}}
$$

one can obtain by induction that, for $m \geq 1, D^{(m)}$ is a polynomial function in $\varepsilon_{t}, \ldots, \varepsilon_{t}^{(m-1)}, \frac{1}{\varepsilon(t-D)+u^{r}}$ without terms of order 0 or 1 . Therefore, $\pi_{1}$ is directly a polynomial function of the variables $\varepsilon_{t}, \ldots, \varepsilon_{t}^{(n-1)}, \frac{1}{1+D}, \frac{1}{\varepsilon(t-D)+u^{r}}$, which is at least quadratic in the variables $\varepsilon_{t}, \ldots, \varepsilon_{t}^{(n-1)}$. Finally, define

$$
c \ell\left(t, \varepsilon_{t}, \ldots, \varepsilon_{t}^{(n-1)}\right)=\pi_{1}\left(\dot{D}, \ldots, D^{(n)}, \varepsilon_{t}, \ldots, \varepsilon_{t}^{(n-1)}, \frac{1}{1+\dot{D}}\right)
$$

Observing that

$$
\frac{1}{1+\dot{D}}=\frac{\varepsilon(t-D)+u^{r}}{2 \varepsilon(t-D)-\varepsilon(t)+u^{r}}
$$

and because $\pi_{1}$ is at least quadratic, it is possible to properly define a neighborhood of the origin $\Omega=[-\underline{\omega}, \bar{\omega}]$ such that

$$
\left|\pi_{1}\left(\varepsilon_{t}, \ldots, \varepsilon_{t}^{(n-1)}\right)\right| \leq c_{\max } \max \left|E_{t}\right|
$$


for $\left|E_{t}\right|$ having values into $\Omega$ (and such that $1+\dot{D}(t)>0$ ). This neighborhood depends on the functional $\ell$ and on the constant $c_{\max }$ and therefore on the feedback gain $K$. Consequently, for $E_{t}([-\bar{D}, 0]) \subset \Omega$, one gets

$$
\left|c \ell\left(t, \varepsilon_{t}, \ldots, \varepsilon_{t}^{(n-1)}\right)\right| \leq c_{\max } \max \left|E_{t}\right|
$$

Therefore, Lemma 2 guarantees the existence of $r(K)>0$ and $\gamma \geq 0$ such that

$$
\forall t \geq 0, \quad|E(t)| \leq r(K) \max \left|E_{0}\right| e^{-\gamma t}
$$

as long as the actuator $\phi$ is not saturated and as (15) applies. Yet, one can observe that a sufficient condition to make sure that the actuator is not saturated is $|\varepsilon(t)| \leq u^{r}-\underline{u}, t \geq 0$. Therefore, by choosing

$$
\begin{aligned}
& \max \left|E_{0}\right| \\
& \leq \frac{1}{r(K)} \min \left\{\frac{\underline{u} \Delta^{*}(|K|)}{2}, u^{r}-\underline{u}, \underline{\omega}(K), \bar{\omega}(K)\right\} \triangleq \theta(K)
\end{aligned}
$$

one can ensure together that this condition is fulfilled for any $t \geq 0$, that the initial condition lies in the neighborhood $\Omega$ and that $|E(t)| \leq \frac{u \Delta^{*}(|K|)}{2}, t \geq 0$. In particular, the condition (10) is also fulfilled.

Finally, the choice $\max \left|E_{0}\right| \leq \theta(K)$ can be expressed in terms of $\Theta$. This gives the conclusion.

The proof of Theorem 2 directly follows from Lemma 4 .

\section{ILlustrative EXAMPLE}

In this section, for illustration purposes, we consider the following simple unstable second-order plant

$$
\left\{\begin{array}{l}
\ddot{x}-\dot{x}+x=\phi(t-D(t)) \\
\int_{t-D(t)}^{t} \phi(s) d s=1, \quad \phi(t)=\operatorname{Sat}_{[0.01,+\infty[}(u(t))
\end{array}\right.
$$

Following the proposed methodology, we introduce the dynamics matrices

$$
A=\left(\begin{array}{cc}
0 & 1 \\
-1 & 1
\end{array}\right), \quad B=\left(\begin{array}{l}
0 \\
1
\end{array}\right)
$$

and implement the control law defined through (7)-(8).

Simulation results for two different feedback gains, respectively $K=[-1-2]$ and $K=[-3-1.5]$ (both yielding a Hurwitz matrix $A+B K$ ), are pictured in Fig.2. Initial conditions are similar, with $x(0)=1, \dot{x}(0)=1$ and $u(t)=1$, $t \in[-\bar{D}, 0]$, resulting into an initial delay $D(0)=1$ the effects of which can be noticed on the beginning of the state response. The control aims at stabilizing the plant toward the target equilibrium $x^{r}=1.1$.

The current delay is calculated by a simple procedure, exploiting the fact that $D \mapsto \int_{t-D}^{t} \phi(s) d s$ is a $\mathcal{K}_{\infty}$ class function. Indeed, numerically, one can evaluate the value of this function for increasing delay values, starting with $D=0$, until reaching the value 1 (which exists as $\phi$ is strictly lowerbounded).
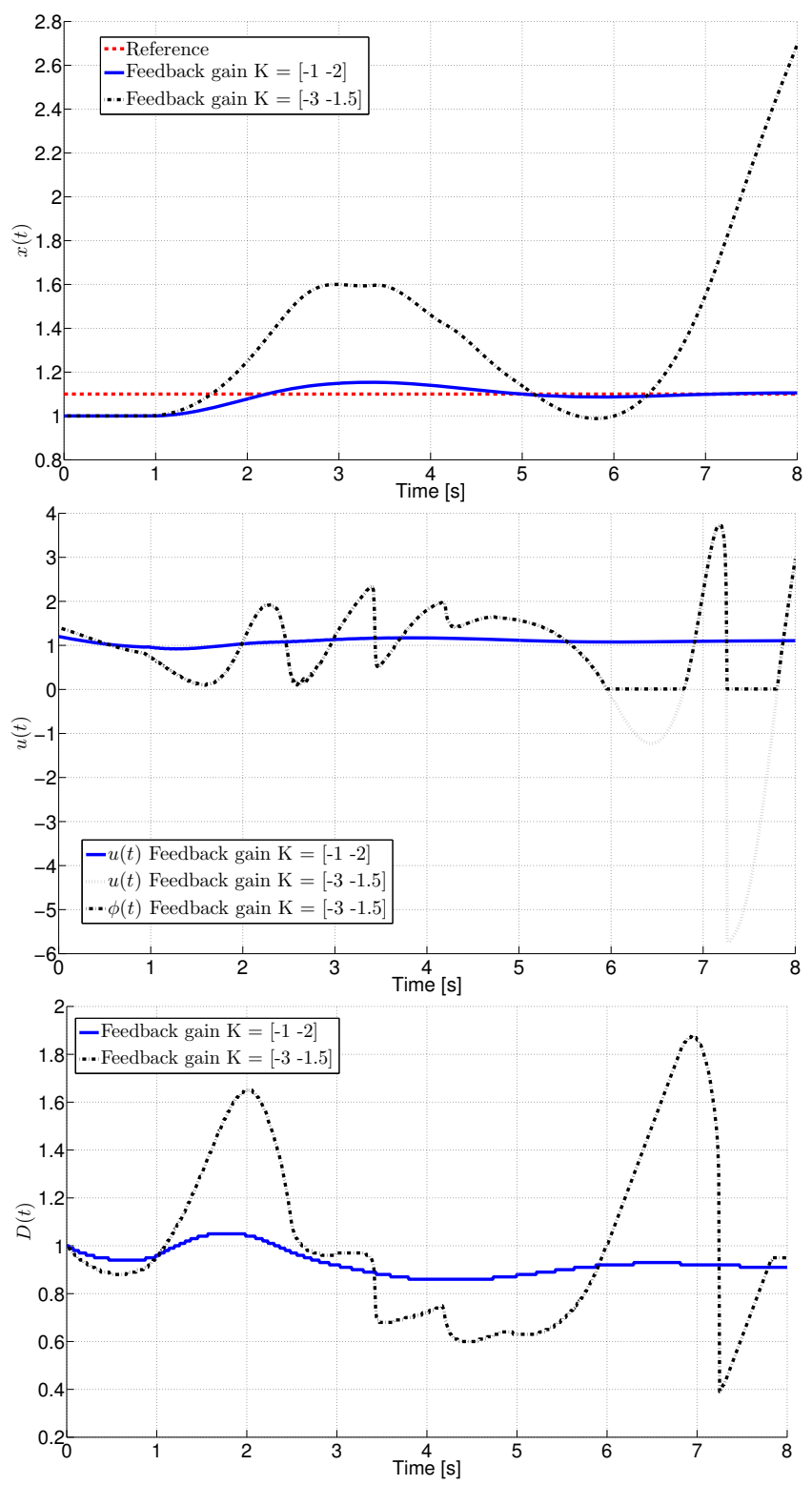

Fig. 2. Simulation results for two feedback gains, respectively $K=$ $[-1-2]$ and $K=[-3-1.5]:$ the state $x$ (top), the control (center) and the corresponding delay (bottom).

Clearly, one can observe that the first controller setting achieves the goal, while the second one does not. This can be reasonably interpreted as a consequence of the gain magnitude as, for the first one, $|K|=2.24$ while, for the second one, $|K|=3.35$. Correspondingly, in Fig.2, the variations of the delay for the second gain setting are larger and larger, which causes the instability.

For comparison, a simple proportional controller with the same feedback gain $K=[-1-2]$ was implemented but does not achieve stabilization and yields the same kind of instability. For sake of readability of the figures, results are not reported here. Yet, this fact enhances the interest of a prediction-based control design. 


\section{CONCLUSION}

In this paper, we have addressed the problem of robust delay compensation of a linear system driven by a delayed input, for a particular class of input-dependent delay (through an integral relation), representative of a large number of processes involving transport of material. The proposed approach is based on a two-steps methodology, which, first, requires the delay variations to be bounded and, in turn, relates these variations to input fluctuations. The obtained sufficient conditions put limits on the initial conditions in accordance with the feedback gain magnitude.

A natural extension of this work would be to study the compliance of the considered framework with state observer design. Other delay defining equations could be considered as-well. Besides, quantitative comparison of the practical performances of the proposed approach with the ones of a prediction-based controller using the (constant) final reference delay $D=1 / u^{r}$ is the natural next step of this approach.

\section{APPENDIX}

\section{A. Proof of Lemma 3}

Before starting the actual proof, we establish preliminary results. In the following, we note $Z=X-X^{r}$ the state tracking error which satisfies, following (6) and (8),

$$
\left\{\begin{array}{l}
\dot{Z}(t)=A Z(t)+B \varepsilon(t-D(t)) \\
\varepsilon(t)=K\left[e^{A D(t)} Z(t)+\int_{t-D(t)}^{t} e^{A(t-s)} B \varepsilon(s) d s\right]
\end{array}\right.
$$

when the actuator is not saturated over the whole time interval $[t-D(t), t]$.

\section{1) Preliminary results:}

Lemma 5: If the actuator is unsaturated over the interval $[t-D(t), t]$, the control variable stated in (8) satisfies the following differential equations

$$
\text { for } \begin{aligned}
1 & \leq m \leq n, \quad \varepsilon^{(m)}-\sum_{l=1}^{m} K A^{l-1} B \varepsilon^{(m-l)}=f_{Z}^{m}(t)+f_{\varepsilon}^{m}(t) \\
& +(1+\dot{D})^{m} K e^{A D} A^{m} Z+K \int_{t-D}^{t} A^{m} e^{A(t-s)} B \varepsilon(s) d s
\end{aligned}
$$

with

$$
\begin{aligned}
& \left\{\begin{array}{l}
f_{Z}^{1}(t)=0 \text { and for } 2 \leq m \leq n \\
f_{Z}^{m}(t)=\frac{d\left[(1+\dot{D})^{m-1}\right]}{d t} K e^{A D} A^{m-1} Z+\frac{d}{d t}\left(f_{Z}^{m-1}(t)\right)
\end{array}\right. \\
& \left\{\begin{aligned}
f_{\mathcal{\varepsilon}}^{1}(t)= & \dot{D} K e^{A D} B \varepsilon(t-D) \text { and for } 2 \leq m \leq n \\
f_{\mathcal{\varepsilon}}^{m}(t)= & \frac{d}{d t}\left(f_{\varepsilon}^{m-1}(t)\right) \\
& +\left[(1+\dot{D})^{m-1}-(1-\dot{D})\right] \varepsilon(t-D) K e^{A D} A^{m-1} B
\end{aligned}\right.
\end{aligned}
$$

Proof: The result is constructively obtained by induction and successive substitutions.

Initial step: one directly gets, taking a time-derivative of the control law (18) and using (17),

$$
\begin{aligned}
\dot{\varepsilon}(t)= & K B \varepsilon(t)+(1+\dot{D}) K e^{A D} A Z+\overbrace{\dot{D} K e^{A D} B \varepsilon(t-D)}^{t}+\overbrace{f_{Z}^{1}(t)}^{=0} \\
& +K \int_{t-D}^{t} A e^{A(t-s)} B \varepsilon(s) d s
\end{aligned}
$$

which gives (19) for $m=1$.

Induction: assume that the property is true for a given $m \geq 1$. We now show that it also holds for $m+1$. Taking a timederivative of (19) for some $m \geq 1$ yields

$$
\begin{aligned}
& \varepsilon^{(m+1)}-\sum_{l=1}^{m} K A^{l-1} B \varepsilon^{(m+1-l)}=\frac{d}{d t}\left(f_{\varepsilon}^{m}(t)\right)+ \\
& \underbrace{\frac{d}{d t}\left(f_{Z}^{m}(t)\right)+\frac{d(1+\dot{D})^{m}}{d t} K e^{A D} A^{m} Z}_{=f_{Z}^{m+1}(t)}+\dot{D}(1+\dot{D})^{m} K e^{A D} A^{m+1} Z \\
& +(1+\dot{D})^{m} K e^{A D} A^{m}[A Z+B \varepsilon(t-D)]+K A^{m} B \varepsilon(t) \\
& -(1-\dot{D}) K e^{A D} A^{m} B \varepsilon(t-D)+K \int_{t-D}^{t} A^{m+1} e^{A(t-s)} B \varepsilon(s) d s
\end{aligned}
$$

Rearranging terms, one obtains (19) for $m+1$. This gives the conclusion.

Further, the sequences $\left(f_{\mathcal{E}}^{m}\right)$ and $\left(f_{Z}^{m}\right)$ satisfy the following properties.

Lemma 6: For $2 \leq m \leq n, f_{\varepsilon}^{m}$ is a polynomial function in $\varepsilon_{t}, \ldots, \varepsilon_{t}^{(m-1)}, \dot{D}, \ldots, D^{(m)}$ without constant nor first-order terms.

Proof: The proof is straightforwardly obtained using the definition of $\left(f_{\mathcal{\varepsilon}}^{m}\right)$ in Lemma 5 together with the fact that

$$
(1+\dot{D})^{m-1}-(1-\dot{D})=\sum_{l=1}^{m-1}\left(\begin{array}{c}
n \\
l
\end{array}\right) \dot{D}^{l}+\dot{D}
$$

which contains no degree 0 terms.

Lemma 7: Assume that $1+\dot{D}>0$. For $2 \leq m \leq n, f_{Z}^{m}$ is a polynomial function in $\varepsilon_{t}, \ldots, \varepsilon_{t}^{(m-1)}, \dot{D}, \ldots D^{(m)}$ and $\frac{1}{1+\dot{D}}$, at least quadratic in the variables $\varepsilon_{t}, \ldots, \varepsilon_{t}^{(m-1)}, \dot{D}, \ldots D^{(m)}$.

Proof: Again, we reason by induction.

Induction: we assume that the property is true for a given $m \geq 2$. Then, using (19) for $m$, one obtains

$$
\begin{aligned}
f_{Z}^{m+1}(t)= & \frac{d\left[(1+\dot{D})^{m}\right]}{d t} K e^{A D} A^{m} Z+\frac{d}{d t}\left(f_{Z}^{m}(t)\right) \\
= & \frac{m \ddot{D}}{1+\dot{D}}\left[\varepsilon^{(m)}-\sum_{l=1}^{m} K A^{l-1} \varepsilon^{(m-l)}-f_{Z}^{m}(t)-f_{\varepsilon}^{m+1}(t)\right. \\
& \left.-K \int_{t-D}^{t} A^{m} e^{A(t-s)} B \varepsilon(s) d s\right]+\frac{d}{d t}\left(f_{Z}^{m}(t)\right)
\end{aligned}
$$

Further, using the induction assumption jointly with the previous lemma, one can conclude that $f_{Z}^{m+1}$ is a polynomial function in $\varepsilon_{t}, \ldots, \varepsilon_{t}^{(m)}, \dot{D}, \ldots, D^{(m+1)}, \frac{1}{1+D}$, at least quadratic in $\varepsilon_{t}, \ldots, \varepsilon_{t}^{(m)}, \dot{D}, \ldots, D^{(m+1)}$.

Initial step: the same arguments as above apply for $m=2$.

2) Design of (15) based on Lemma 5, 6 and 7: As the dynamics matrix that we consider in (3) is of companion type, Cayley-Hamilton theorem gives

$$
A^{n}=-\sum_{i=0}^{n-1} a_{i} A^{i}
$$


Therefore, for $m=n$ (19) simply gives

$$
\begin{aligned}
\varepsilon^{(n)} & -\sum_{l=1}^{n} K A^{l-1} B \varepsilon^{(n-l)}=f_{Z}^{n}(t)+f_{\mathcal{\varepsilon}}^{n}(t) \\
& -\sum_{m=0}^{n-1} a_{m}\left[(1+\dot{D})^{n} K e^{A D} A^{m} Z+K \int_{t-D}^{t} A^{m} e^{A(t-s)} B \varepsilon(s) d s\right]
\end{aligned}
$$

or again, using now (19) for $m$ ranging from 1 to $n-1$

$$
\begin{aligned}
\varepsilon^{(n)} & -\sum_{l=1}^{n} K A^{l-1} B \varepsilon^{(n-l)}=f_{Z}^{n}(t)+f_{\mathcal{\varepsilon}}^{n}(t) \\
& -\sum_{m=0}^{n-1} a_{m}(1+\dot{D})^{n-m}\left[\varepsilon^{(m)}-\sum_{l=1}^{m} K A^{l-1} B \varepsilon^{(m-l)}\right. \\
& \left.-f_{Z}^{m}(t)-f_{\mathcal{\varepsilon}}^{m}(t)-K \int_{t-D}^{t} A^{m} e^{A(t-s)} B \varepsilon(s) d s\right] \\
& -\sum_{m=1}^{n-1} a_{m} K \int_{t-D}^{t} A^{m} e^{A(t-s)} B \varepsilon(s) d s \\
& -a_{0}\left[(1+\dot{D})^{n} K e^{A D} Z+K \int_{t-D}^{t} e^{A(t-s)} B \varepsilon(s) d s\right]
\end{aligned}
$$

Besides, using the Leibniz formula,

$$
(1+\dot{D})^{n-m}=1+\sum_{l=1}^{n-m}\left(\begin{array}{c}
n-m \\
l
\end{array}\right) \dot{D}^{l}
$$

and the expression of (18), one can define

$$
\begin{aligned}
\pi_{1}\left(\dot{D}, \ldots, D^{(n)}, \varepsilon_{t}, \ldots, \varepsilon_{t}^{(n-1)}\right) \triangleq & f_{Z}^{n}(t)+f_{\varepsilon}^{n}(t) \\
& +\sum_{m=0}^{n-1} a_{m}(1+\dot{D})^{n-m}\left(f_{Z}^{m}(t)-f_{\varepsilon}^{m}(t)\right) \\
& -\sum_{m=1}^{n-1} a_{m}\left[\sum_{l=1}^{n-m}\left(\begin{array}{c}
n-m \\
l
\end{array}\right) \dot{D}^{l}\right] \\
& \times\left[\varepsilon^{(m)}-\sum_{l=1}^{m} K A^{l-1} B \varepsilon^{(m-l)}-K \int_{t-D}^{t} A^{m} e^{A(t-s)} B \varepsilon(s) d s\right] \\
& -a_{0} \sum_{l=1}^{n}\left(\begin{array}{c}
n \\
l
\end{array}\right) \dot{D}^{l}\left[\varepsilon(t)-K \int_{t-D}^{t} e^{A(t-s)} B \varepsilon(s) d s\right]
\end{aligned}
$$

From there, one can obtain the dynamic (15) by observing that

$$
\begin{aligned}
\sum_{l=1}^{n} K A^{l-1} B \varepsilon^{(n-l)} & +\sum_{m=1}^{n-1} \sum_{l=1}^{m} a_{m} K A^{l-1} B \varepsilon^{(m-l)} \\
& =-\sum_{l=1}^{n} b_{0} k_{n-l} \varepsilon^{(n-l)}
\end{aligned}
$$

which is proven in the next section. Further, from the definition (20) of $\pi_{1}$, using Lemma 6 and $7, \pi_{1}$ is a polynomial in the variables $\varepsilon_{t}, \ldots, \varepsilon_{t}^{(n-1)}, \dot{D}, \ldots, D^{(n)}$ and $\frac{1}{1+\dot{D}}$, at least quadratic in the variables $\varepsilon_{t}, \ldots, \varepsilon_{t}^{(n-1)}, \dot{D}, \ldots, D^{(n)}$. This gives the desired result.

\section{B. Proof of (21) using companion matrix properties}

One can reformulate the term under consideration in (21) as follows

$$
\sum_{l=1}^{n} K A^{l-1} B \varepsilon^{(n-l)}+\sum_{m=1}^{n-1} \sum_{l=1}^{m} a_{m} K A^{l-1} B \varepsilon^{(m-l)}
$$

$$
\begin{aligned}
& =\sum_{l=1}^{n} K A^{l-1} B \varepsilon^{(n-l)}+\sum_{p=2}^{n} \sum_{m=n-1-p}^{n-1} a_{m} K A^{p-n+m-1} B \varepsilon^{(n-p)} \\
& =K B \varepsilon^{(n-1)}+\sum_{l=2}^{n}\left[K A^{l-1} B+\sum_{m=n-1-l}^{n-1} a_{m} K A^{l-n+m-1} B\right] \varepsilon^{(n-l)}
\end{aligned}
$$

To study the second term in this last expression, consider $m_{j}^{i}$ the $j^{\text {th }}$ coefficient of $A^{i} B$. As $A$ is a companion-type matrix, one gets

$$
m_{j}^{i}=\left\{\begin{array}{cl}
0 & \text { if } j \leq n-i-1 \\
m_{j+1}^{i-1} & \text { if } n-i \leq j \leq n-1 \\
-\sum_{l=n-i+1}^{n} a_{j-1} m_{j}^{i-1} & \text { if } j=n
\end{array}\right.
$$

and that

for $i \in \mathbb{N}, 1 \leq j \leq n$ and $1-j \leq p \leq \max \{i, n-j\}$

$$
m_{i}^{j}=m_{j+p}^{i-p}
$$

Then, the coefficient of the $(n-p)^{t h}$ derivative can be rewritten as follows

$$
\begin{aligned}
& K A^{l-1} B+\sum_{m=n-1-p}^{n-1} a_{m} K A^{p-n+m-1} B \\
& =K A^{l-1} B+\sum_{i=1}^{l-1} a_{n+i-l} K A^{i-1} B \\
& =-k_{n-l} m_{n-l+1}^{l-1}-\sum_{j=n-l+2}^{n} k_{j-1}\left[m_{j}^{l-1}+\sum_{i=n-j+1}^{l-1} a_{n+i-l} m_{j}^{i-1}\right]
\end{aligned}
$$

We now prove by induction that, for $l \geq 0$ and for $n-l+2 \leq j \leq n, m_{j}^{l-1}=-\sum_{i=n-j+1}^{l-1} a_{n+i-l} m_{j}^{i-1}$.

For $l=2$ and $j=n$, the proposition is indeed true as $m_{j}^{l-1}=-a_{n-1} b_{0}$ and $-\sum_{i=n-j+1}^{l-1} a_{n+i-l} m_{j}^{i-1}=-a_{n-1} m_{n}^{0}$. Now, assume that the property is true for a given $l \geq 2$ and for all integer $j n-l+2 \leq j \leq n$. Consider $j$ such that $n-l+2 \leq j \leq n-1$, then

$$
\begin{aligned}
\sum_{i=n-j+1}^{l} a_{n+i-l-1} m_{j}^{i-1} & =\sum_{i=n-j}^{l-1} a_{n+i-l} m_{j}^{i} \\
& =\sum_{i=n-j}^{l-1} a_{n+i-l} m_{j+1}^{i-1} \\
& =-m_{j+1}^{l-1}=-m_{j}^{l}
\end{aligned}
$$

using (22) and the induction assumption. This gives the desired result for $n-l+2 \leq j \leq n-1$. For $j=n$, one gets using successively (23) and (22)

$$
\begin{aligned}
\sum_{i=1}^{l} a_{n+i-l-1} m_{n}^{i-1} & =\sum_{i=1}^{l} a_{n+i-l-1} m_{i}^{n-1} \\
& =\sum_{i=n+1-l}^{n} a_{j-1} m_{j}^{l-1}=-m_{n}^{l}
\end{aligned}
$$

which gives the conclusion. Therefore, for $2 \leq l \leq n$,

$$
\begin{aligned}
K A^{l-1} B+\sum_{m=n-1-p}^{n-1} a_{m} K A^{p-n+m-1} B & =-k_{n-l} m_{n-l+1}^{l-1} \\
& =-k_{n-l} m_{n}^{0}=-k_{n-l} b_{0}
\end{aligned}
$$

and $K B=-k_{n-1} b_{0}$. This concludes the proof of (21). 


\section{REFERENCES}

[1] Z. Artstein. Linear systems with delayed controls: a reduction. IEEE Transactions on Automatic Control, 27(4):869-879, 1982.

[2] N. Bekiaris-Liberis and M. Krstic. Compensation of state-dependent input delay for nonlinear systems. In Conference on Decision and Control, 2011.

[3] D. Bresch-Pietri, J. Chauvin, and N. Petit. Adaptive control scheme for uncertain time-delay systems. Automatica, 48:1536-1552, 2012.

[4] D. Bresch-Pietri, J. Chauvin, and N. Petit. Invoking Halanay inequality to conclude on closed-loop stability of a process with input-varying delay. In 10th IFAC Workshop on Time Delay Systems, 2012.

[5] D. Bresch-Pietri, J. Chauvin, and N. Petit. Prediction-based feedback control of a class of processes with input-varying delay. In Proc. of the American Control Conference, 2012.

[6] M. Chèbre, Y. Creff, and N. Petit. Feedback control and optimization for the production of commercial fuels by blending. Journal of Process Control, 20(4):441-451, 2010.

[7] C. Depcik and D. Assanis. One-dimensional automotive catalyst modeling. Progress in energy and combustion science, 31(4):308369, 2005.

[8] K. Gu and S. I. Niculescu. Survey on recent results in the stability and control of time-delay systems. Journal of Dynamic Systems, Measurement, and Control, 125:158, 2003.

[9] A. Halanay. Differential equations: stability, oscillations, time lags, volume 23. Academic Press, 1966.

[10] J. Harmand and D. Dochain. The optimal design of two interconnected (bio) chemical reactors revisited. Computers \& chemical engineering, 30(1):70-82, 2005.

[11] A. Ivanov, E. Liz, and S. Trofimchuk. Halanay inequality, yorke $3 / 2$ stability criterion, and differential equations with maxima. Tohoku Mathematical Journal, 54(2):277-295, 2002.

[12] M. Jankovic. Recursive predictor design for linear systems with time delay. In American Control Conference, 2008, pages 4904-4909. IEEE, 2008.

[13] M. Krstic. Boundary control of PDEs: a course on backstepping designs. Society for Industrial and Applied Mathematics Philadelphia, PA, USA, 2008.

[14] M. Krstic. Delay compensation for nonlinear, adaptive, and PDE systems. Birkhauser, 2009.

[15] A. Manitius and A. Olbrot. Finite spectrum assignment problem for systems with delays. IEEE Transactions on Automatic Control, 24(4):541-552, 1979.

[16] W. Michiels and S. I. Niculescu. Stability and Stabilization of TimeDelay Systems. Society for Industrial and Applied Mathematics, 2007.

[17] Y. S. Moon, P. G. Park, and W. H. Kwon. Robust stabilization of uncertain input-delayed systems using reduction method. Automatica, 37(2):307-312, 2001.

[18] M. T. Nihtila. Finite pole assignment for systems with time-varying input delays. In Proc. of the 30th IEEE Conference on Decision and Control, pages 927-928, 1991.

[19] R. H. Perry, D. W. Green, and J. O. Maloney. Perry's chemical engineers' handbook, volume 7. McGraw-Hill New York, 1984.

[20] N. Petit, Y. Creff, and P. Rouchon. Motion planning for two classes of nonlinear systems with delays depending on the control. In Proceedings of the 37th IEEE Conference on Decision and Control, pages 1007-1011, 1998 .

[21] J.-P. Richard. Time-delay systems: an overview of some recent advances and open problems. Automatica, 39(10):1667-1694, 2003.

[22] M. Sbarciog, R. De Keyser, S. Cristea, and C. De Prada. Nonlinear Predictive Control of processes with variable time delay. A temperature control case study. In Proc. of the IEEE International Conference on Control Applications, pages 1001-1006. IEEE, 2008.

[23] O. J. M. Smith. A controller to overcome dead time. ISA Journal, 6(2):28-33, 1959.

[24] E. Witrant. Stabilisation des systèmes commandés par réseaux. $\mathrm{PhD}$ thesis, Laboratoire d'Automatique de Grenoble, 2005.

[25] D. Yue and Q. L. Han. Delayed feedback control of uncertain systems with time-varying input delay. Automatica, 41(2):233-240, 2005. 